\title{
SHIELDING MEASUREMENTS OF THE SPACE SHUTTLE (PART I - MEETING THE TEST CHALLENGE)
}

\author{
Buzz Brezinski \\ Diane Kempf \\ Naval Air Warfare Center \\ Patuxent River, Maryland
}

\author{
Robert Scully \\ NASA Johnson Space Center \\ Houston, Texas
}

\begin{abstract}
The Naval Air Warfare Center (NAWC) at Pax River teamed with the National Institute of Standards and Technology (NIST) to perform a shielding test of the NASA Space Shuttle Orbiter "Endeavour." Pax River was responsible for testing in the high frequency range up to 18 GHz. NASA imposed some very tight limitations on the time allotted to perform the test and the amount of power that could be radiated during the performance of the test. This required Pax River to perform much development work on our existing shielding effectiveness test method using reverberation techniques in order to meet NASA's needs. This paper discusses the method in detail and describes the development work and its validation.
\end{abstract}

\section{Background}

Most military and commercial EMI limits were developed with sound engineering and a measure of conservative guessing which sometimes results in over design and added weight. This translates to higher costs for design, materials, and production. Present market trends are pushing for design to the platform and knowing the environment where you will "plug in". To accomplish such objectives, it is imperative to characterize the shielding effectiveness of the platform. This paper discusses the 4.2 to $18 \mathrm{GHz}$ frequency range that NAWC Pax River was responsible for in a joint venture with NASA and the National Institute of Standards and Technology (NIST) to test the NASA Space Shuttle. NIST was primarily responsible for the lower frequencies, but also provided consultation for the higher frequency range. The methodology is a progression of ideas developed by NAWC, the Naval Surface Warfare Center (NSWC), NIST, and NASA (See references 1, 2, 3, and 4). The changing avionics marketplace is relying on increased partnerships such as this to meld industry expertise and realize elevated goals. This "endeavour" is a case study of a truly unique EMI after-the-fact avionics-related evaluation in a new world of doing business.

Once developed, and modified to meet NASA's tight requirements on the amount of power that could be radiated, and the amount of time allotted to perform the test, the shielding test methodology was first evaluated in a "worst case" chamber (NAWC Patuxent River shielded hangar) on a shielding artifact and on an F-18 aircraft prior to testing of the NASA spacecraft. The measurements agreed closely with other shielding data taken of a "holey artifact" in various test environments. The hangar at the Shuttle Landing Facility at Kennedy Space Center served as the "reverberation chamber" for the spacecraft. Pax River's method is a greatly modified version of the traditional nested reverberation chambers method. Our method utilizes spatial sampling and frequency "stirring" in order to obtain large quantities of data very efficiently. Frequency stirring was used to simulate the physical boundary movement of the RF standing wave patterns within the spacecraft and to augment the spatial sampling externally. Averaging about a frequency window was also used to stabilize data with fewer samples than would be necessary with peak determination. The method has been shown to be quite accurate and repeatable. This presentation discusses the details of the test method, software development, and testing of the artifact and of the F-18. Also, the software used to automate the measurements will be described in detail. This was essential to maximizing the accuracy and efficiency of the measurements.

\section{Test Methodology}

Testing was performed using a state of the art version of reverberation methods. Traditionally, a reverberant shielding effectiveness test is performed using "nested reverberation chambers." The field in each chamber is "stirred" using an electrically large metallic tuner that is capable of suitable displacement at the lower frequency range of the test. Statistically, this "stirring" produces a very uniform field over a revolution of the tuner. Since it is physically impractical to construct and locate a tuner that would be large enough to be effective to use in a chamber of the size needed, this test uses several antennas placed around the aircraft to provide spatial sampling instead of displacement sampling (reference 3). Another array of antennas is placed inside the aircraft that are moved to various locations for internal spatial sampling. Frequency averaging, or electronic stirring, is used to simulate the physical boundary movement of the RF standing wave patterns and augment the statistical sampling in the cavity 
(reference 2). As in the traditional methods, the received levels are compared to those received when the aircraft is not present (the reference measurements). The difference is the shielding effectiveness of the aircraft. A big advantage of this method is that it avoids the inaccuracies associated with conventional direct radiation methods caused by: standing wave patterns, radiation aspect angle, and antenna factor errors. These usually render very inaccurate and unrepeatable measurements. Reverberant technology has been demonstrated to be statistically highly repeatable and uniform over various reverberant environments.

\section{Software}

All data was gathered with the NAWC EMI Laboratory's Aircraft Shielding Effectiveness Software, written with HP VEE software. Simplistically, the software controls a matrix of transmit and receive signals of any size to the present limitations of 6 X 6 (6 transmit antennas and 6 receive antennas). The number of receives is a result of the number of spectrum analyzers available. The transmit antennas are controlled using an IEEE-488 bus controlled RF high power switcher. Each transmit and receive can be uniquely identified, either with a name or a number. The software allows input for all associated calibration and test factor files; transmit cables, receive cables, pre-amplifiers, switches, and an auxiliary transmit factor, and noise floor information. Either linear or log stepped scans are available. Each frequency step defines a processing frequency window or "frequency stir span". The stir span is a frequency band that is approximately a half step to the left of the center frequency step to a half step to the right of the frequency step. Within this band are the "frequency stir steps". In this case, the software scanned the frequency range from $4.2 \mathrm{GHz}$ to $18 \mathrm{GHz}$ at $100 \mathrm{MHz}$ steps. The stir step size was $5 \mathrm{MHz}$ within the $100 \mathrm{MHz}$ window. Data gathered across this processing window was averaged. Typically, peak levels are gathered during reverberation, but NAWC opted for averaging. Averaging tends to be much more statistically repeatable with fewer test samples and converges much more quickly than peak data. Upon entering each frequency step stir cycle, power was leveled to a predetermined user input value. All data was normalized to this input power (essentially an insertion loss measurement). This also allowed for flexibility of adjusting power anytime during the test process to optimize dynamic range and assure preamplifiers/amplifiers were not driven to compression. This feature also allowed data processing of the files of different power sets without need for additional compensation. In additional to all the raw corrected data, there were two types of processed data; a peak data file and an average data file. This allowed real time monitoring of "apparent" shielding data updated after each complete frequency stir measurement cycle consisting of frequency stirring with all transmit antennas. All data was normalized to a "default reference measurement" for this real time viewing. Several laboratory software packages were used in post data processing; a multiple file merge, multiple file averaging, multiple file peak processing, and a multiple file Chi square arrangement algorithm. This assisted in merging multiple files to the final processed values and to examine data distributions to assure statistical conformity and uniformity. The multi-file averaging algorithm was the primary software for final Orbiter data processing. This post processing performs the linear average on all the files identified.

\section{Test Validation-An Evolution}

Existing NAWC software was heavily modified to incorporate the particulars for the Orbiter and the nonideal test environment, as well as to improve accuracy and efficiency to meet limitations on the test time and the field levels associated with the Orbiter. In a relatively ideal chamber, trial runs were performed using a $3 \times 3$ test matrix (3 transmit and 3 receive antennas) to examine all aspects of the software and to assure it was operating correctly. Various frequency windows, step rates, stir step rates were examined to determine suitability for the project. A $100 \mathrm{MHz}$ step fixed rate and a $5 \mathrm{MHz}$ fixed stir step were chosen for overall efficiency, accuracy, and ability to identify resonant structures with the largest possible averaging window. Averaging proved to converge many times faster than the peak values (See Figure 1) as shown for a typical shielding test and even with a limited data set (120 independent samples). A typical peak test requires thousands of samples to achieve a reliable expected value. However, averaging is at the expense of about 20 $\mathrm{dB}$ of dynamic range. Thus, high gain, low noise preamplifiers were incorporated to offset the dynamic range loss of averaging. Individual matrix elements (individual $\mathrm{X}, \mathrm{Y}$ : transmit, and receive files) tracked very closely with only the frequency averaging. This is demonstrated in Figure 2. The standard deviation of individual matrix elements was predominantly within a $2-2.5 \mathrm{~dB}$ window. 


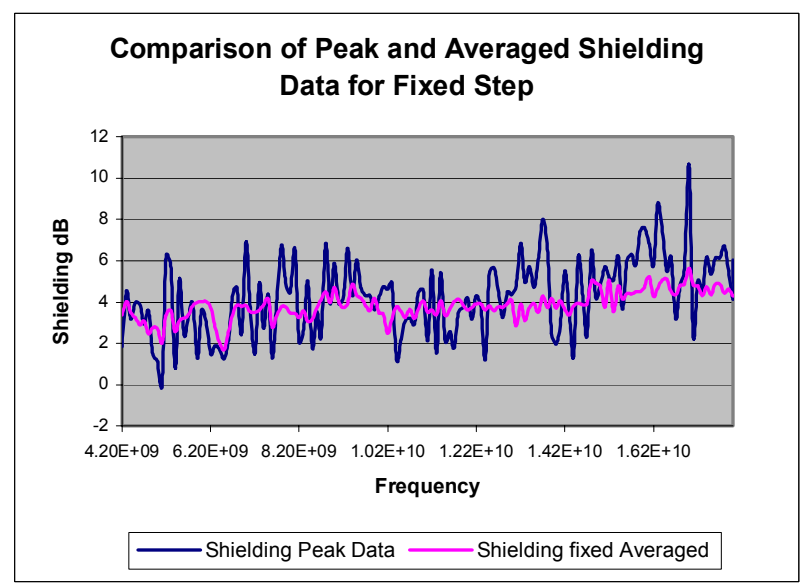

Figure 1. One Test File Comparison of Peak and Average

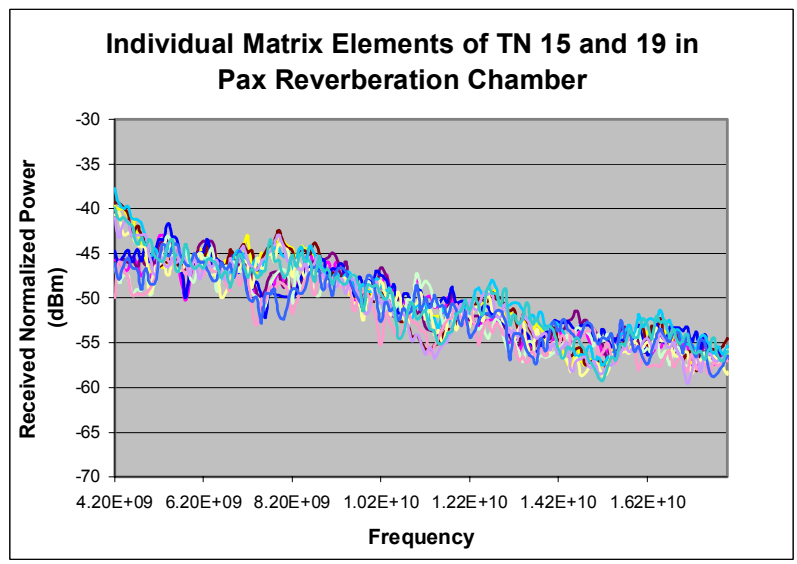

Figure 2. Data Elements of Two Different Tests

Typically, the larger the averaging frequency window, the smoother the results. Testing conducted with frequency windows approaching $\lambda / 4$ demonstrated even tighter response, however, such large windowing averages wash out cavity resonant effects. Averaging the individual matrix elements together further enhances stability as demonstrated by Figure 3 where three different tests are compared. The receive antennas from one test to another were moved and run on different days. Here the individual matrix elements from a test are averaged and compared to other averaged received power data from two other tests. The three independent test sets demonstrate very good agreement.

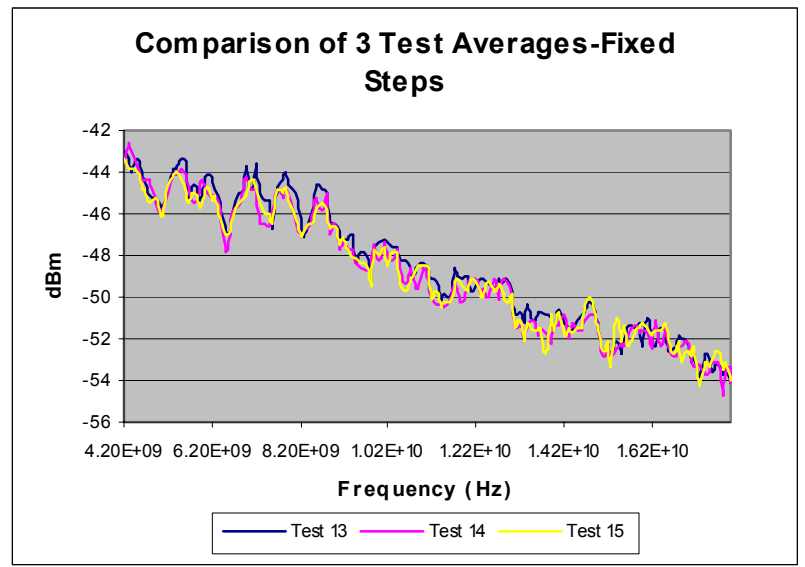

Figure 3. Averaged Data Tracking Over 3
Independent Tests

The statistical data distribution is a good indicator of the integrity of a reverberation chamber. For received power in a reverberation chamber, this is characterized with the $\mathrm{Chi}^{2}$ cumulative probability distribution with 2 degrees of freedom. Plots of an $18 \mathrm{GHz}$ distribution over two sets of test data are demonstrated in Figure 4. These are plotted against the ideal $\mathrm{Chi}^{2}$ cumulative probability distribution. The distribution tracks the ideal very well, even though three different makeshift antennas (with somewhat different responses) were used to obtain the data. Throughout this study, the lower end distributions $(4.2 \mathrm{GHz})$ were also plotted. However, these typically traced the ideal well, so they are not included. The 18 $\mathrm{GHz}$ distributions tended to reveal more information about the integrity of the chamber and of the data gathered.

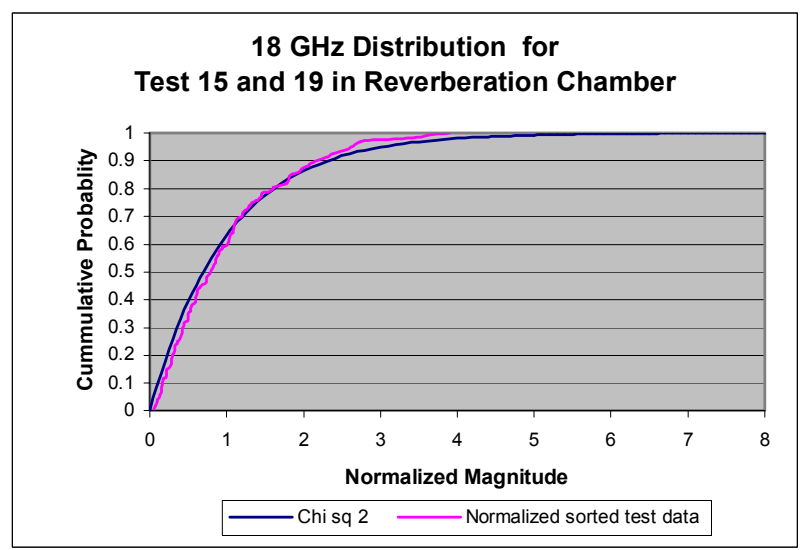

Figure 4. 18 GHz Cumulative Probability of the NAWC Reverberation Chamber 


\section{An EMI Space of a Different Kind-World's Largest Reverberation Chamber?}

A reverberation chamber of a size to support the Endeavour is simply not available. However in the past, NAWC had experimented and performed reverberation evaluations in their Aircraft Shielded Hangar with good results (references 1 and 4). An evaluation in this hangar would provide the opportunity to evaluate a worst-case scenario, as the hangar is heavily loaded with a number of wooden structures, aircraft and large panels of anechoic material. Luckily, a Navy aircraft project provided an opportunity for this "small scale Endeavour", an excellent adaptation of resources to accomplish test objectives in a changing business place. Prior to the aircraft arrival, various reference tests were conducted in numerous locations about the hangar. Each test consisted of a $3 \times 4$ matrix of transmit and receive antennas. Immediately apparent, the data distributions of the individual $\mathrm{x}, \mathrm{y}$ matrix elements were much more widely spaced than was experienced in the "more ideal" reverberation chamber. This was likely due to the greater wave travel times and more prominent mass absorption due to multiple bounces when antennas were aimed towards the far walls (curved sides) of the hangar where buildings and absorber material were located. The stronger signals were merely more direct received signals. These signals encountered less space loss and less absorption by hangar contents. Figure 5 demonstrates the individual elements of two tests in the hangar showing the range of data, which had a standard deviation of typically within $5 \mathrm{~dB}$.

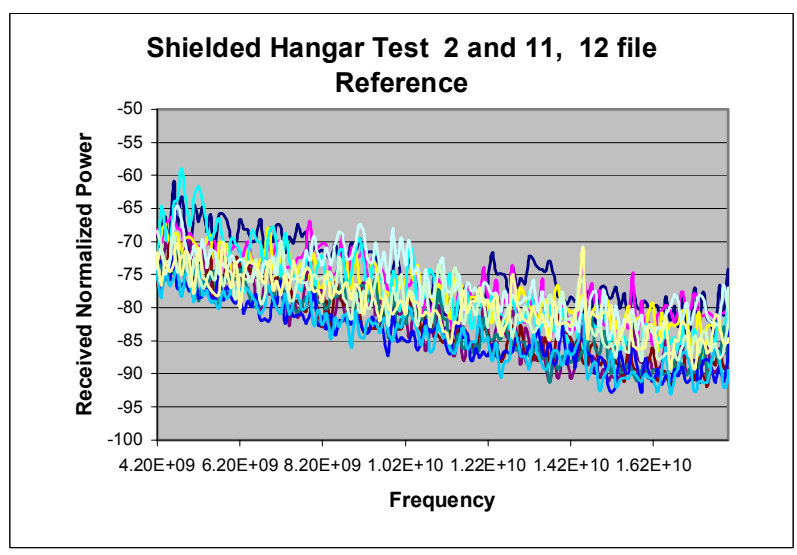

\section{Figure 5. Data Elements of Two Different Tests in Shielded Hangar}

Figures 6 demonstrates the statistical $\mathrm{Chi}^{2}$ distribution of these tests for $18 \mathrm{GHz}$. As expected, there was a bit of departure from ideal where the variations in path lengths begin to distort the $18 \mathrm{GHz}$ waveform.

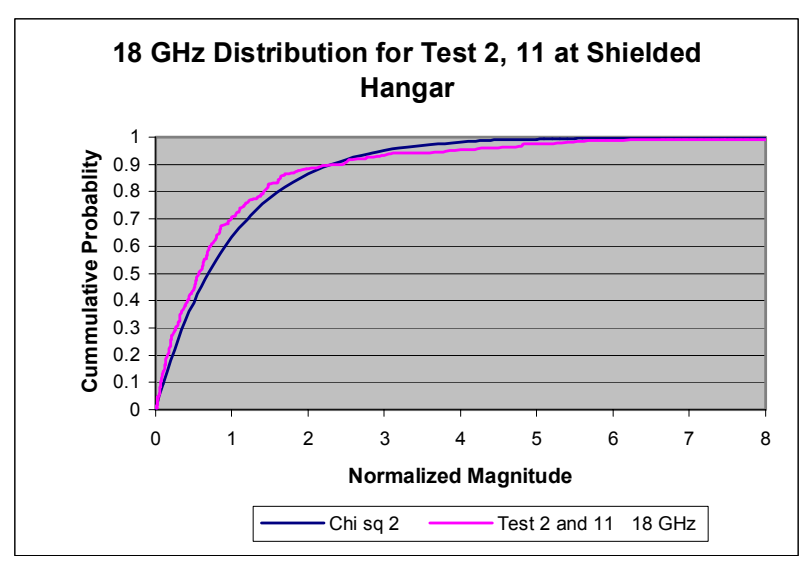
Figure 6. 18 GHz Cumulative Probability
Distribution With Wire Antennas

Up to this point, evaluations in the Pax River Shielded Hangar were conducted with monopole transmit antennas fabricated by stripping the shield and connector from one end of a high frequency RF cable, an adaptation of available resources. The use of double ridge-guided horns was anticipated with the Orbiter testing, so an evaluation was conducted with these. Figure 7 demonstrates the individual elements of two tests of data taken using horns in the hangar showing the range of frequency-averaged data.

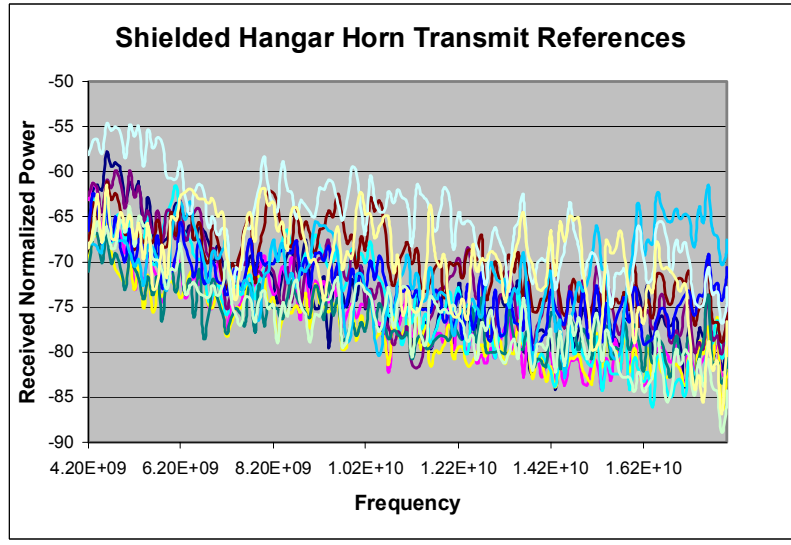

\section{Figure 7. Data Elements of Two Different Tests in Shielded Hangar with Horns}

The standard deviation of these data was typically within $5 \mathrm{~dB}$, but some values well exceeded that value, a considerable departure from an ideal chamber. Clearly, it was not as uniform as the wire antennas. Figure 8 demonstrates the statistical distributions of these tests for $18 \mathrm{GHz}$. The horn antenna response demonstrates a wider deviation of the data and 
distortion of the $18 \mathrm{GHz}$ distribution than the wire antenna. This is possibly due to excessive losses in areas of the hangar due to wooden buildings, absorber material, etc. and very good reflections elsewhere. A non-directional antenna may provide some statistical smoothing. In a typical room sized reverberation chamber at frequencies below $1 \mathrm{GHz}$ (reference 1), it has been shown that there is very little difference between a directional and non-directional antenna, but above that, the direct and the reflected signal become very different due to the greater absorption of materials in the reverberation chamber. However, these findings of the omni directional antenna would appear to contradict the requirement for a directional antenna in a typical reverberation chamber. This is most likely the result of the widely varying path lengths and the resultant displacement of the individual elements of the test matrix (each transmit/receive element), i.e. the signal must travel quite a bit further in a large chamber to establish the reverberation modal structure sufficiently. Early reflections are a bit like being in the direct beam width in a smaller chamber and are very easily encountered, but hard to predict. The omni directional antenna radiates energy in many directions, which appears to reduce/break up the effect of the varying path lengths in establishing the reverberation modal structure. However, care must still be taken to avoid direct coupling.

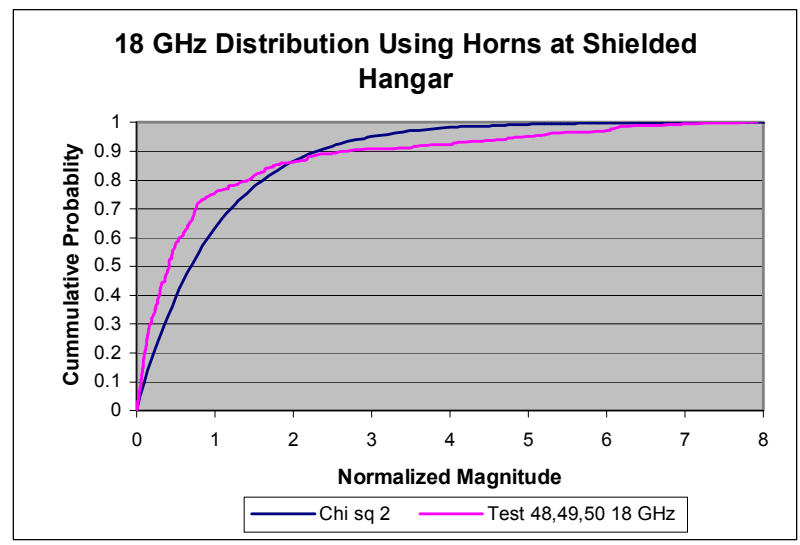

Figure 8.18 GHz Cumulative Probability Distribution With Horn Antennas

\section{The Holey Artifact and a Valid Space}

The poor distribution of the data using horns required additional evaluation of the "worst case" chamber. To demonstrate the final validity of test results for this methodology, NAWC was able to obtain a shielding artifact from NIST and demonstrated that this method gives the same results as those using the traditional nested chamber method in a reverberation chamber. The shielding artifact is a metal box, developed jointly by NIST and NSWC. It is an aluminum enclosure, measuring $0.73 \mathrm{~m} \mathrm{X} 0.93 \mathrm{~m} \mathrm{X} 1.03 \mathrm{~m}$, with a tuner inside. Each of the six sides contains five randomly drilled circular holes, $1.6 \mathrm{~cm}$ in diameter, to provide internal to external coupling. NAWC performed the shielding test in the large Shielded Hangar. To test the artifact, NIST used the nested chamber method in their reverberation chamber to take extensive data. Even with using only one location for the receive antennas in the hangar and three tuner positions inside the NIST box, giving a total of just 12 data sets ( 240 samples) for each frequency, the shielding values agreed very closely with those of NIST, verifying the method with the proven, accepted, traditional reverberation method. Figure 9 shows the shielding of the artifact box. The NIST measurements are the peak values recorded for each frequency. This shows the variability of the NIST data with frequency before averaging. Figure 9 also shows a 21 period moving average of the NIST measurements, and the NAWC measurements. Even with what would be considered a very limited data set, the curve of the NAWC data follows that of the average NIST data quite closely. So, in spite of the non-ideal standard deviation and distribution of the data in that Shielded Hangar, the results were excellent. However, great care should be taken to avoid high bias early reflections. The averaging process appears to provide consistency in spite of a nonideal distribution.

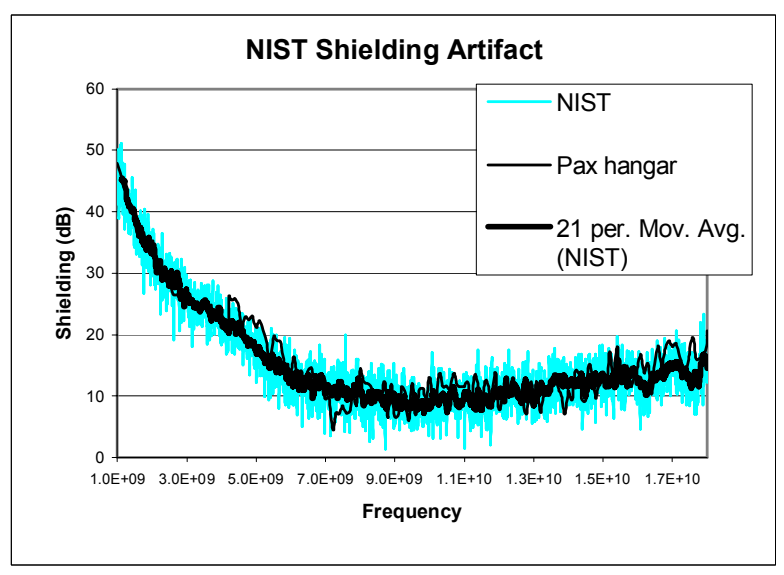

Figure 9. Shielding of the NIST Artifact

\section{Conclusions}

New methodologies are needed to conduct more realistic EMI evaluations of avionics equipments in this new, rapidly changing business environment. EMI spaces and practices do not necessarily need to conform to the usual paradigms. Teamwork and innovation paid 
off big dividends in achieving success with this large "Endeavour".

When viable to use, average reverberation test data converges much faster and is much more tolerant of test environment than peak data gathering. Care is required to avoid dynamic range issues using averaging.

The test method of frequency stirring in a reverberant environment has been shown to be accurate compared to the nested chamber method in a reverberation chamber.

Testing in the large SLF Hangar and the Pax River Shielded Hangar has shown that there are test phenomenons unique to very large reverberation chambers that need further investigation.

\section{Acknowledgements}

The PAX River shielding test team would like to thank the entire NIST test team: Bob Johnk, David Navotny, Chris Grosvener, Nino Canales, and Dennis Camell for their insights, suggestions, and expertise. It was a pleasure working with them all. Also of NIST, we especially acknowledge Galen Koepke. With his vast experience and knowledge of reverberation methods, Galen provided valuable advice and was an excellent liaison between the NIST and Pax test teams, using two very different test methods to measure the shielding of the Orbiter. All of the folks at NASA, too numerous to name here, who coordinated the effort, supported the Orbiter, and provided assistance when needed, are all greatly appreciated. We heartily thank our Team who fully supported the "Endeavour" by getting equipment together, organizing and delivering that equipment and filling the gaps in workload back home during the "Endeavour". And last, but certainly not least, a big thanks to Bob Scully of NASA, a true leader who facilitated this Endeavour in spite of all the odds. We are grateful to be part of such a successful, interesting, project.

\section{References}

1. Kempf, Diane R., Kenneth “Buzz” Brezinski, October 2001, Using Reverberation Techniques for Testing an Intercommunications System on a Navy Aircraft (2001: A Test Lunacy), 2001 IEEE Digital Avionics Conference

2. Grosvenor, Chriss. A., Robert T. Johnk, David R. Novotny, Nino Canales, Claude M. Weil, Jason Veneman, January 2003, A Two-Phase Airframe Shielding Performance Study Using Ultra-wideband Measurements Systems, NISTIR 6622, National Institute of Standards and Technology

3. Hatfield, Michael, April 2003, "In-Situ Shielding Measurements", Reverberation Chamber, Anechoic Chamber, OATS, and GTEM User's Meeting

4. Kempf, Diane R., Kenneth "Buzz" Brezinski, September 2002, Shielding Effectiveness Testing of the Learjet 45, NAWC AD EMI Report

5. Ladbury, John M.; Galen H. Koepke, June 1999, Reverberation Chamber Relationships: Corrections and Improvements or Three Wrongs Can (Almost) Make a Right, Reverberation Chamber, Anechoic Chamber, and OATS User's Meeting 